\title{
Taxonomy of fibre mat misalignments in pultruded GFRP bridge decks
}

\author{
M. Poulton ${ }^{1}$, W.M. Sebastian ${ }^{1, *}$ \\ ${ }^{1}$ Department of Civil, Environmental and Geomatic Engineering, University College London, \\ Chadwick Building, Gower Street, London, WC1E 6BT, UK
}

\section{*Corresponding authoremail address: w.sebastian@ucl.ac.uk}

Abstract: This paper presents a taxonomy of the fibre mat misalignments found in a multicelled pultruded GFRP deck, based on high-resolution images of the polished cross sections. A dual approach to misalignment taxonomy is presented, one based on misalignment morphology, the other grounded in the manufacturing provenance of the misalignments. Each misalignment is characterised using, alternately, a Gaussian function, the angle to its steepest tangent, its length and its height. Some scatter in the type and severity of the misalignments was found within the web-flange junctions. The deck's top flange was found to be thicker in regions containing a double-backed or flipped mat layer, which was probably due to displacement of the internal mandrels during pultrusion. A previously verified analytical approach based on classical laminate theory predicts that, owing to stress concentrations, the present Gaussian misalignments lead to a 57\% knockdown in tensile load capacity.

\section{Keywords: Glass fibres, fabrics/textiles, defects, pultrusion}

\section{Introduction}

Glass fibre reinforced polymer (GFRP) cellular bridge decks offer numerous advantages compared to conventional steel or reinforced concrete alternatives. The cellular design and high stiffnessand strength-to-weight ratio of GFRP enables prefabricated decks to be quickly lifted onto the abutments using low capacity cranes, which minimises traffic disruption [1]. The modular design is eminently suited to increasing the load capacity of existing bridges through dead weight reduction. Also, the superior corrosion resistance (to de-icing salts in particular) can reduce the maintenance and inspection costs over a prolonged design life.

Of the range of manufacturing techniques available, pultrusion has proved popular for GFRP decks as it facilitates the continuous production of standardized, thin walled sections of complex geometries and fibre architectures. The standardization of the geometry and material properties of 
pultruded GFRP sections has been crucial to their adoption in bridge decks and in civil engineering structures more widely. Thus, any discrepancies that exist between the assumed material properties and those of the as-built deck must be properly understood.

To that end, this paper investigates a key source of material variability in pultruded decks; namely, misalignment of the fibre mats. As shown in Figure 1(a), the fibre mats in a GFRP deck are often randomly misaligned in the transverse (normal-to pultrusion) direction. The term "fibre waviness" is commonly used to describe this type of defect [2]. However, for pultruded decks the trajectory of the mats can take various forms. Hence, throughout this paper the term misalignment is used to refer to any deviation of the mats from their optimal location; that is, the location that maximises the mechanical properties of the deck when subject to realistic in-service loads.

Fibre mat misalignments are only problematic if their adverse effects are not accounted for at the design stage. There are three reasons why this is often the case for GFRP decks:

1. The mat misalignments arise due to variabilities in the pultrusion process, and so their location and severity are highly random.

2. The mechanical properties of the deck provided by the manufacturer are established from standard tests on flat coupons taken from the webs and flanges. However, the properties of the flats are not representative of the web-flange junctions (WFJs) due in part to the presence of mat misalignments and other defects (including voids and resin-rich zones) that are unique to the junctions.

It should be noted that, given the definition of misalignment provided above, even in the absence of the random misalignments that arise during the pultrusion process, the fibre mats may still be considered misaligned if they do not produce the optimal deck performance. This becomes an important consideration at the junctions, which are subject to complex, multi-directional stress states due to the local contact pressure distribution exerted by heavy lorry tyres [3]. The positioning of fibre mats that produces the optimal resistance to these loads is difficult to determine and may not be consistent with the positioning specified by the manufacturer. Furthermore, manufacturers of GFRP decks do not typically disclose detailed information regarding the 'intended' fibre architecture and mat positioning. 

mat misalignments in pultruded decks. For example, the US standard ASTM D4385-19 [4] states that misalignments are permissible if the section achieves the material properties stated by the manufacturer, which is inadequate for the reasons outlined in point 2 above. The European standard EN 13706-2 [5] outlines a defect state in pultruded sections as "An unintentional or unspecified misalignment of mat or fabric reinforcing material in relation to the contour of a pultruded section", which is limited to an outof-plane deviation of $1.5 \mathrm{~mm}$ (or $20 \%$ of the part thickness). However, as outlined in the paragraph above, in areas of complex geometry the 'contours' of the GFRP deck are ill-defined and may not describe the optimal trajectory for the fibre mats. Thus, the specified limits on mat misalignment should be relative to the performance of the deck.

A lack of clear guidance regarding the acceptable positioning of the fibre mats has resulted in many previous studies reporting on misaligned fibre mats in pultruded GFRP decks. For example, Coogler et al. [6] described "misoriented" and "wavy" fibre mats in the WFJs of a section of Duraspan deck. For the same decking system, Yanes-Armas et al. [7] described "wrinkling" of the inner mats, of the mats through-the-thickness of the web. These misalignments were inconsistent between two nominally identical WFJs. Xin et al. [8] commented on mat "folding" in the WFJs of a cellular GFRP deck, which was described as more pronounced for the WFJs with thicker webs, flanges and fillet radii. In a recent study by Sa et al. [9], Figure 3 of their paper clearly shows misalignment of the mats passing presented a first attempt at measuring the fibre mat "waviness" in a unit of Fiberline ASSET deck, by manually plotting points along each fibre mat layer on a transparent plastic sheet using a sharp-pointed marker.

Aside from bridge decks, several other studies have demonstrated similar mat misalignments in standard pultruded sections. For example, misalignments in both the flats [10], free ends [11] and WFJs [12] [13] of I-sections are reported to produce significant variability in the local buckling loads, likewise for box beams [14]. This demonstrates the prominence of mat misalignments as a generic issue in pultruded sections. However, beyond these initial observations, no data that relate the severity of mat misalignment to the properties of the pultruded section have been produced. Indeed, a search through 
Mottram's constantly renewed database of over 2700 publications on research and development with pultruded profiles [15] shows that the present paper is the first to address this important issue.

Despite this paucity of data, the influence of variable mat misalignments on the failure of pultruded sections is evidenced by the fracture patterns that develop during failure. The effect is most pronounced at key junctions within the deck that exhibit significant misalignments. As shown in Figure 1(b), failure of the WFJs manifests as delaminations along the misaligned mat layers and kinking at the steepest tangent angle, which is accompanied by transverse cracks that occasionally propagate to the surface. These surface cracks create paths for water ingress, which can further deteriorate the fibre-toresin bond strengths. A comprehensive summary of the figures in previous studies that show similar fractures within the WFJs of both pultruded GFRP decks and I-sections is provided in Table 1 of Sebastian et al. [2].

A complete understanding of fibre mat misalignments starts with the pultrusion process. To that end, Figure 2 shows an illustration of the pultrusion process, where the key stages that influence the positioning of the mats are emphasized (in bold). The specific process details for a given pultruded section vary and are typically undisclosed by the manufacturer, so the illustration is based on generic information provided in Shaw-Stewart and Sumerack [16] and Meyer [17]. A description of the key stages is given below.

During pultrusion, layers of fibre mats are pulled and progressively formed from their initial planar shape into that of the final section using a series of metal or high-density polyethylene forming guides. Rovings are pulled in a similar manner through a series of guiding holes and inserted as discrete layers between the mats. This process (referred to as reinforcement collimation) is more complex for cellular sections as the fibre mats must be completely wrapped around an internal mandrel in order to form the hollow shape. This wrapping method is preferable to inserting separate flat mats into each of the webs and flanges, which does not achieve continuity of the mats through the web-flange junctions (WFJs).

Thick, multi-celled pultruded sections with multiple layers of fibre mats and rovings - as used in GFRP bridge decks - require many forming guides and mandrels that afford only approximate control over the positioning of the fibres. Lateral shifting and crowding of the rovings prior to die entry can misalign the surrounding mats, and wrinkles in the mats can also arise during the wrapping process. 
115 Within the die, compression of the fibre rovings and impregnation of the resin using die-injection can

116 result in further movement of the fibres, producing wrinkles and resin-rich zones near the surface of the

117 section. For cellular sections, transverse gaps or overlaps in the mats are inevitable due to the wrapping

118 process, but their location and extent are subject to variability during manufacture.

As a result, the cross section of the cured profile can exhibit multiple types of misalignment that occur randomly and simultaneously throughout the section, as illustrated in the insert to the bottom right of Figure 2. It is important to distinguish between these different types, as they may have a differing impact on the structural performance of the GFRP deck. Unlike the previous studies listed above that use terms such as "misoriented", "wavy", "wrinkled" and "folded" interchangeably, the study reported on in the rest of this paper defines multiple categories of mat misalignment based on their morphology and origin in manufacture.

The issue of fibre misalignments has been studied extensively in the context of aerospace- and automotive-grade FRPs [18]. In this case, experimental investigations typically involve inducing a localised misalignment in a small flat coupon under lab conditions, enabling the severity to be precisely controlled and quantified. For example, the effect of wrinkles in CFRP prepregs has been investigated by inserting either steel rods [19], aluminium wire [20], or prepreg strips [21] transversely across the laminate. Potter et al. [22] built on these studies by developing a taxonomy of defect states in prepreg composite components, highlighting that a significant proportion of misalignments are influenced predominantly by design decisions, including the choice of corner radii and part curvature, rather than by errors in the manufacturing process itself.

The studies mentioned in the preceding paragraph produce misalignments in flat FRP coupons that are easily characterised using simple sinusoidal functions, which enables theoretical prediction of the laminate stiffness and strength reduction as a function of the wave height and amplitude. Bogetti et al. [23] applied 2D piecewise classical laminate theory to a cross-ply laminate with a half sine-wave middle layer. This method was later shown by Hsiao and Daniel to correlate well with experimentally obtained stiffness [24] and strength [25] values for carbon- and glass-FRP coupons under uniaxial compression. El-

141 Hajjar and Peterson [19] used the method to accurately predict the stiffness of unidirectional carbon-FRP laminates with wrinkles described using a graded Gaussian function. It is now prudent to transfer the above concepts to the mat misalignments found in as-manufactured pultruded GFRP bridge decks. 
From the issues outlined above, the objectives of this paper are as follows, namely to:

- Develop a suitable technique for imaging and measuring the transverse fibre mat misalignments within a pultruded GFRP bridge deck.

- Present a taxonomy of fibre mat misalignment based on morphology and origin in manufacture.

- Quantify the inconsistency of misalignments observed in nominally identical WFJs of the deck.

- Assess whether the fibre mat misalignments can be characterised using simple mathematical functions that can be implemented into an analytical model to predict the reduction in mechanical properties of the deck.

A specific pultruded bridge deck has been used throughout this paper to illustrate the above objectives. The mat misalignments observed in the bridge deck used in this study are broadly representative of those found in other commercial pultruded, cellular bridge decks to date. Similar misalignment morphologies to those presented in this paper can be seen in previously published crosssectional images of a variety of bridge decks from different manufacturers (for example [7], [8] and [9]) and from a larger decking system from the same manufacturer [2]. Thus, the imaging and characterisation technique proposed in this paper can be applied to a wide range of pultruded, cellular decks.

The scope of this paper is limited to mat misalignments in the transverse-to-pultrusion direction, as can be seen in the cut cross-sections of the deck. It is noted that the tensile force applied to the fibre mats during pultrusion should minimise misalignments in the longitudinal direction.

The following section outlines the imaging technique used and gives details of the different fibre mats observed in the chosen deck specimens. The taxonomy of fibre mat misalignments is presented in Section 4, which is then used in Section 5 to compare the WFJs from each of the five specimens. Section 6 presents the analytical analysis, which is followed by conclusions and recommendations for future work.

\section{Methodology}


Figure 3(a) shows the geometry of the GFRP decking system used in this study, which is a rectangular unit that encloses two diagonal stiffeners to form three internal cells. The short sides of the deck have complementary tongue-and-groove connectors to facilitate adhesive bonding. The GFRP material consists of E-glass fibres embedded in an isophthalic polyester resin. Specific details of the fibre mats and rovings used in the deck are provided in Section 3.2. The deck geometry was optimised to satisfy the traffic loading in the Dutch standard NEN 6788: 1995 [26] and was manufactured by Fiberline using resin injection within the die. The decking system has been used in several bridges throughout Europe, including a $99 \mathrm{~m}$ cyclist and pedestrian bridge in Reinbek, Germany in 2009. In this and other bridges, the deck has spanned transversely between longitudinal girders with the thicker flange on top to provide adequate resistance to local tyre loads.

Five panels of bridge deck were selected for analysis based on differences in the type of mat misalignments observed within the cross section of the deck, indicating that the panels were produced in different batches. These were labelled specimens A to E, where specimen A exhibited the fewest misalignments and $\mathrm{E}$ the most. A comparison of the misalignments within the WFJs of each specimen is provided in Section 5 of this paper.

A single $80 \mathrm{~mm}$ wide section of deck was cut from each panel, and the cut surface was then deburred and polished using progressively finer sandpaper up to 600-grit. The application of an oil-based substance to the cut surface of the deck increased the reflectivity of the $0^{\circ}$ fibres, thus enhancing the contrast between the rovings and mats. Petroleum jelly was used for this purpose as it did not fade significantly with time.

The cross sections from each specimen were imaged using a Canon 7D DSLR camera with a telephoto zoom lens that had a minimum focal length of $200 \mathrm{~mm}$ at maximum magnification, as shown in Figure 3(b). At this distance, the image window was approximately $80 \mathrm{~mm}$ by $53 \mathrm{~mm}(2 / 3$ aspect ratio) with a resolution of $3456 \times 5184$ pixels. Through trial and error, it was found that placing a light source at a $45^{\circ}$ angle above the cut surface produced maximum contrast between the roving and mat layers.

For each specimen, images were taken of the four WFJs at the ends of the diagonal stiffeners, named top left (TL), top right (TR), bottom left (BR) and bottom right (BR) as can be seen in Figure 3(a). 
Centring the image at each of these junctions provided sufficient coverage of the adjoining 'flats' of the deck and any additional misalignments that occurred in the webs or flanges.

The images were post-processed in ImageJ, a freely available image editing software that enables scaling, transformation and measurements of images. First, each image was scaled using the estimated pixels/mm obtained from scaling the horizontal ruler aligned with the top surface of the deck. Then, the images were rotated and translated to ensure the top deck surface was perfectly horizontal and at a fixed distance from the edge of the image.

The distortion effects from the lens were checked by taking an image of a $4 \mathrm{~mm}$ square grid and measuring the displacement of the vertices relative to a uniform grid overlaid onto the image in postprocessing. The image showed a minimal degree of pincushion distortion, with a maximum corner displacement of $0.2 \mathrm{~mm}$. In the centre of the image (where the web-flange junction was located) this distortion was negligible.

Once scaled, the trajectory of each mat could be traced by placing markers every $0.25 \mathrm{~mm}$ along the top and bottom edges of the fibre mat layers. The misalignment of the layer was evaluated from the midline of the top and bottom markers. The uncertainty with regards to the placement of the markers was influenced by the geometry of the fibre mats, as outlined in the following section.

\subsection{Geometry of fibre mats}

The cross-sectional images of the WFJs revealed the geometry and layup of the fibre mats and rovings used within the bridge deck panels. As shown in Figure 4(a), the GFRP deck comprised a combination of biaxial, triaxial and complex stitched mats either side of a large core of textured rovings. The biaxial mats appear as a thick white layer $\left(90^{\circ}\right.$ bundles $)$ with a row of blue ellipses $\left(0^{\circ}\right.$ bundles $)$ that have areas of pure white resin in the gaps between them. The triaxial mats have a similar cross-sectional appearance to the biaxial, as the $+45^{\circ}$ and $-45^{\circ}$ bundles appear as a single white layer, with an adjacent row of blue ellipses. Note that these mats were inserted either with the $0^{\circ}$ bundles above or below the offaxis bundles. The third type of fibre mat present in the deck was a thin complex mat, shown to the right of Figure 4(a), which had a combination of small $0^{\circ} / 90^{\circ}$ woven bundles and randomly orientated fibres. A surface veil also lined the outer perimeter of the deck. 

straight and non-uniformly distributed fibres. Detailed micro-CT images of the fibres within a pultruded roving core showing a similar macro-structure can be found in a recent study by Baran et al. [27]. markers along the top and bottom interfaces of the off-axis bundles (white bands). The fibre mat-toroving interface was very clear, and the markers could be placed with an error of less than $0.08 \mathrm{~mm}$ (the height of 5 pixels). However, marking the fibre mat-to-fibre mat interface required occasional interpolation through the small areas of pure resin that were present due to the gaps between the $0^{\circ}$ bundles. that can be used as a reference for the fibre mat misalignments reported on in the rest of this paper. This idealisation is shown in Figure 4(b), which also gives a magnified view of the WFJ in the insert at the top of the figure. As the manufacturer's intended fibre architecture is not disclosed, the idealised section assumes the mats are parallel with the contours of the profile, as is consistent with EN 13706-2 [5]. (black) and a biaxial mat (red). The internal cells are lined with a veil, biaxial and triaxial mat (blue), which wrap completely around the cells. In the top flange, an additional horizontal biaxial mat runs

\section{Taxonomy}

Figure 5 presents a summary of the different categories of mat misalignments observed within the GFRP bridge deck cross-sections. As shown, six categories are defined based on the morphology of the misalignment, where the name given to each category refers to a distinct shape as follows:

1. Double-Back (DB): a mat layer returns upon itself. 
2. Flip (F): at a web-flange junction, the end of a mat layer is flipped to the other side of the junction.

3. Corner Wrinkle (CW): a localised loop in the corner of a mat layer.

4. Near-Surface Wrinkle (NSW): a localised dip in the mat layers near the surface of the specimen.

5. Waviness (W): a pronounced undulation of a mat layer, generally as it passes between layers of roving.

6. Drop (D): a mat layer that drops down due to the termination of the layer below.

A detailed description of each category is provided in the following sub-sections. An example image is provided for each category. The top of each figure containing the image indicates where on the deck cross section and from which specimen (A to E) the image was taken. Within each figure a schematic of the fibre mat trajectories is provided for clarity. Note, that when discussing a single WFJ, the terms 'closed side' and 'open side' refer to the adjoining flanges that form acute and obtuse angles with the web, respectively.

\subsection{Double-back}

An extreme example of mat misalignment is a double-back, which is caused by errors during the mat wrapping process of cellular profiles. Figure 6 shows an example of this feature; note that the schematic diagram shows a larger portion of the top flange than the image below. It is seen that the two internal fibre mats did not follow their intended path along the top flange of the deck. Rather, near the local midpoint of the flange the two layers simultaneously double-backed, and again for a second time at the centre of the WFJ before terminating on the closed side of the junction. It is only this second double back that is seen in the example image given in the figure. The biaxial mat layer that ran continuously along the top flange was forced up and over these double-backed layers. Consequently, the WFJ was highly asymmetric; the closed side of the junction had more than double the intended number of fibre mat layers, which then also significantly increased the thickness of that side of the junction from $9.0 \mathrm{~mm}$ to $10.5 \mathrm{~mm}$ (due to movement of the mandrel, as explained in Section 5.3). 
A mat flip is a class of misalignment that occurred only within the WFJs where the end of a mat layer was flipped over from one side of the junction to another, as shown in Figure 7. In this example, the end of the triaxial mat layer on the closed side of the junction was flipped over and terminated on the open side, rather than traverse around the fillet radius as intended. The flipped layer pushed up the biaxial mat layer on the open side of the junction, which then dropped down a steep gradient within the junction before rising again as it entered the closed side. Another side-effect was a triangular-shaped area of pure resin that formed in the gap between the flipped triaxial mat and the biaxial mat above. Shrinkage of the pure-resin area during curing created a void at the centre of the WFJ, as seen in the example image. The flipping of the mat is also a consequence of errors in the collimation process.

\subsection{Corner wrinkle}

A corner wrinkle is a localised loop in a mat layer, typically manifesting at the corner of one of the internal mats that was wrapped around the mandrel during pultrusion. The example shown in Figure 8 occurred in the triaxial mat layer at the bottom right WFJ of specimen B. Corner wrinkles form during collimation of the mat, as incorrect wrapping of one layer can result in excess width of mat being taken up in the form of a wrinkle, which then gets pushed to the corners of the mat when the fibres are compressed into the die. A pure resin zone also formed in the gap behind the wrinkle.

\subsection{Near-surface wrinkle}

A second type of wrinkling occurred in the mat layers near the outer surfaces of the specimen due to a different stage of the pultrusion process. During resin injection within the die, the flow of the resin through the fibre mats can cause depressions in the outer layers, typically adjacent to the injection ports within the die. The example in Figure 9 shows a surface wrinkle in the top biaxial mat and surface veil, which occurred at the centreline of the WFJ. Note how the height of the wrinkle is greater in the biaxial mat than in the surface veil. This is likely due to the lower porosity of the biaxial mat leading to greater drag forces as the resin passed through the fibres. A pure-resin area filled the 'well' formed by the wrinkle, which could be seen from the outer surface of the specimen. 

misalignment is characterised by fitting a Gaussian function to the coordinates of the layer mid-thickness. The resulting curve-fits are used to compare the WFJs in Section 5, and as the basis for the analytical analysis in Section 6. The Gaussian function is given by the equation:

$$
f(x)=A_{\mathrm{w}} e^{-\frac{x^{2}}{2 c^{2}}}
$$

Where $A_{\mathrm{w}}$ is the amplitude (height) of the wrinkle and $c$ is the horizontal distance between the centre of the curve and the inflection point (point of maximum misalignment angle).

\subsection{Waviness}

Waviness is defined as the gradual undulation of one or more mat layers within the section, in contrast to wrinkles that occur over a finite length of mat. Waviness is a consequence of the non-uniform distribution of rovings either side of the mat, which arises due to lateral shifting or crowding of the rovings during reinforcement collimation.

314 For the GFRP deck used in this study, there is only one layer of rovings within the core of the section. Hence, the only location where a fibre mat passes through the middle of the roving core is at the WFJs. As seen in the example in Figure 10, the rovings within the web were shifted up and to the closed side of the junction, whilst in the flange they were shifted down, creating a misalignment in the biaxial mat layer that was 'intended' to pass horizontally through the junction. As shown later in Section 5, the randomness of roving shifting led to a range of wavy shapes that were inconsistent between the different WFJs. Unlike wrinkles, the morphology of the wavy mat layer within the junction did not lend itself to characterisation using a mathematical function. Instead the severity of waviness was evaluated using the length $\left(L_{\mathrm{w}}\right)$, height $\left(H_{\mathrm{w}}\right)$ and maximum misalignment angle $\left(\theta_{\max , \mathrm{w}}\right)$, as defined in Figure 10.

\subsection{Drops}

A drop refers to a mat layer that is terminated within the section. For cellular sections, the wrapping of the internal mats inherently creates a discontinuity at the point where the ends meet. The guidance in EN 13706-2 [5] specifies a minimum of $5 \mathrm{~mm}$ overlap of the mats for closed sections. 
and waviness of the mat layer which shortens the path length. For the bridge deck used in this study, large gaps between the ends of the two internal mats were observed along the top flange of the deck. These gaps caused pronounced drops in the mat layers above, as shown in Figure 11. The thin complex mat above these layers also terminated immediately before the WFJ. The distance from the junction at which the drops occurred were inconsistent between different specimens. This inconsistency was evaluated by calculating the distance from the WFJ centreline of termination of the biaxial $\left(D_{\mathrm{b}}\right)$, triaxial $\left(D_{\mathrm{t}}\right)$ and complex $\left(D_{\mathrm{c}}\right)$ mat layers, as shown in Figure 11. A comparison of these values for each WFJ is presented in Section 5.

\section{Inconsistency of misalignments}

\subsection{WFJ comparison}

Using the taxonomy of misalignments defined in the previous section, it is now possible to compare the misalignments observed in the five different bridge deck specimens used for this study. This comparison focuses on the area surrounding the internal WFJs on the top side of the deck, as these junctions are directly beneath the applied tyre load. Figure 12(a) to (e) show the top-left and top-right WFJs for each of the five specimens. The junctions are labelled with two letters, the first denoting the specimen designation (A to E) and the second whether the junction was on the left or the right side, for example ' $\mathrm{CL}$ ' for specimen $\mathrm{C}$, left side. On each image, the misaligned mat layers have been identified and the class of misalignment labelled using the coding system defined at the bottom of the figure. Note that in Figure 12(e) the image of junction ER has a discontinuity on the open side of the junction due to a cut made in the specimen prior to imaging for other purposes.

From this figure, the inconsistencies pertaining to each class of misalignment are as follows:

- Double back (DB): the double-back occurred only in EL.

- $\quad$ Flip (F): the flip occurred only in DL, DR and CR. However, the flips in DL and DR were inconsistent. In DL, the flipped mat layer kinks towards to closed side of the junction before flipping over to the open side. Conversely, the flipped mat in DR had almost no kink and flipped immediately to the open side. The kink in DL decreased the area of the triangular zone of pure resin to approximately $4.1 \mathrm{~mm}^{2}$, compared to $10.3 \mathrm{~mm}^{2}$ in DR and $6.7 \mathrm{~mm}^{2}$ in CR. The smaller 
area of pure resin in DL precluded the development of a shrinkage hole that can be seen clearly in the other two flipped junctions.

- Corner wrinkle (CW): as noted in Section 4.3, the corner wrinkle appeared only in the bottom right WFJ of specimen B, and thus is not included in Figure 12.

- Near-surface wrinkles (NSW): specimens A and B had negligible wrinkling of the top mat layers, whereas specimens $\mathrm{C}$ and $\mathrm{D}$ had pronounced wrinkles at the centre of the junction. For comparison, Figure 13 shows the Gaussian function fits of the surface wrinkles at the centre of junctions CL, CR, DL, DR. The Gaussian constants $A_{\text {w }}$ and $c$, along with the $R^{2}$ values for each curve are given in the top right of the figure. It is seen that this function provides a good fit to the wrinkle geometry, with $R^{2}$ values of greater than 0.97 except for CL, which had the smallest wrinkle height and was asymmetric about its peak. The $c$ values (horizontal distance to inflection point of curve) ranged between $4.7 \mathrm{~mm}$ and $5.9 \mathrm{~mm}$ and were largely independent of the wrinkle height. The right WFJs of specimens C and D showed a 135\% and 34\% increase in wrinkle height compared to the left side, respectively.

- Waviness (W): the waviness of the horizontal biaxial mat was highly variable both between the different specimens and within the same specimen in the left and right junctions. The measured length $\left(L_{\mathrm{w}}\right)$, height $\left(H_{\mathrm{w}}\right)$ and steepest tangent angle $\left(\theta_{\max , \mathrm{w}}\right)$ of the wavy profiles are given in Figure 14(a), (b) and (c) respectively, as defined in Section 4.5. The maximum waviness length was $30.52 \mathrm{~mm}$ (for BL) due to the severe shifting of the rovings, which were pushed up and into the closed side of the junction. Compared to BR, this shifting produced a $66 \%, 30 \%$ and $16 \%$ increase in the waviness length, height and angle respectively. The maximum height and tangent angle were $2.9 \mathrm{~mm}$ and $26^{\circ}$, respectively, both of which occurred in CR. However, in this case the increased waviness is primarily a consequence of the flipped layer itself rather than shifting of the rovings.

- Drops (D): the dotted lines labelled ' $\mathrm{D}$ ' in Figure 12 show the drops in the biaxial mat layer that runs across the full width of the top flange due to termination of the three underlying layers (refer back to Figure 11 for a clear illustration of these drops). The distance from the WFJ centreline at which the complex, biaxial and triaxial mats terminated are shown in Figure 15(a), (b) and (c) respectively, but only for the junctions that did not contain a flip or double back. 
Negative distances are on the closed side of the junction and positive on the open side. This figure demonstrates yet again the asymmetry of the junctions, this time both between the left and right WFJs (AL vs AR for example) and on either side of the same junction.

For context, the values of misalignment severity reported above can be compared to analogous misalignment morphologies observed in industrial FRP components manufactured using techniques other than pultrusion. For example, for a CFRP prepreg component, Wang et al. [21] reported a maximum waviness length, height and tangent angle of $28.17 \mathrm{~mm}, 0.50 \mathrm{~mm}$ and $5.8^{\circ}$, respectively. While the waviness length of the CFRP prepreg is comparable to that of the GFRP deck, the maximum height and tangent angle are $480 \%$ and $350 \%$ greater in the GFRP deck, respectively.

In conclusion, the six classes of mat misalignment defined in Section 4 were highly inconsistent between the top WFJs of the deck. This inconsistency was often greater between WFJs from the same deck specimens than between the WFJs from different specimens. As outlined earlier, this is likely due to lack of control over the positioning of the fibre mats during pultrusion, which introduced significant randomness into the observed misalignments.

\section{2 $\quad$ Flange wrinkles}

In addition to the near-surface wrinkles at the centre of the WFJs, significant wrinkling was observed in the top flange of specimen D (labelled DT) and the bottom flange of specimen B (labelled BB), shown in Figure 16(a) and (b) respectively. From the Gaussian curve fits in Figure 16(c), it is seen that these winkles exhibited the maximum and minimum values of amplitude $A_{\mathrm{w}}(2.0 \mathrm{~mm})$ and width $c$ (2.8 $\mathrm{mm}$ ), respectively, when compared to the WFJ wrinkles. That these wrinkles occurred near to the local flange midspan is problematic, as the top mat layer is subject to high stresses that alternate between tensile and compressive as a tyre rolls over the surface of the deck.

The influence of these near-surface wrinkles on the mechanical properties of the top flange are investigated in Section 6 using an analytical model based on classical lamination theory. 
The WFJs also varied with respect to the thicknesses of their adjoining flanges. Figure 17 shows the measured flange thicknesses either side of each WFJ, along with a horizontal line indicating the manufacturers stated flange thickness of $9.0 \mathrm{~mm}$. It is seen that the measured values differ significantly from the intended thickness, are inconsistent between the different WFJs and show a high degree of asymmetry between the open and closed side of the same WFJ. The largest asymmetry occurred in EL, where the closed-side thickness was $16 \%$ larger than that on the open side. That the increased thickness occurred on the side containing the double-backed mat layers suggests that the variable flange thicknesses were a result of displacement of the mandrel during pultrusion.

As illustrated in Figure 18(a) during pultrusion of the decking system used in this study, there are three mandrels that are cantilevered into the die. Within the die, the free ends of the mandrels are subject to compressive forces from the fibre mats and rovings, and from the resin that is injected at high pressure. If these forces are unbalanced around the perimeter of the mandrels, for example due to a double-back or flipped mat layer, then the mandrels may move out of concentric alignment with the die. The severity of mandrel misalignment can be estimated by measuring the wall thicknesses, which was carried out for the left-hand cell of specimen E. The results are shown in Figure 18(b), where the centroid based on the wall thicknesses. It is seen that the double backed layers appear to have rotated the mandrel 
deck. An idealised model of top the flange with a near-surface wrinkle is shown in Figure 19(a). The model comprises a core of rovings sandwiched between two mat layers with $90^{\circ}$ fibres. The top and bottom mat layers are of equal thickness $t_{\mathrm{m}}$. The top mat undergoes a single out-of-plane undulation as described by the Gaussian parameters $A_{\mathrm{w}}$ and $c$ using Eq.(1), with the centroid located at $x=0$.

As shown in Figure 19(b), the local orientation of the wrinkled mat layer (in 1-3 coordinates) rotates an angle $\theta$ with respect to the global $(x-z)$ coordinates of the laminate. The variation of $\theta$ as a function of $x$ is given by the following relation:

$$
\theta(x)=\tan ^{-1}\left(-\frac{A_{\mathrm{w}} x}{c^{2}} e^{\frac{-x^{2}}{2 c^{2}}}\right)
$$

where $A_{\mathrm{w}}$ is the wrinkle amplitude and $c$ the width. Table 1 gives the geometric values used in the analysis, as well as the maximum and minimum values of the Gaussian parameters $A_{\mathrm{w}}$ and $c$ based on the curve fits in Figure 16(c). The length $L$ of the laminate was $30 \mathrm{~mm}$, calculated from the height of the wrinkle at $\pm L / 2$ being less than $1 \%$ of $A_{\mathrm{w}}$ for the maximum wrinkle width $c_{\max }$. This ensured that the stiffnesses were calculated only for the portion of the flange containing the wrinkle. The mat thickness $t_{\mathrm{m}}$ was measured from the cross-sectional images and the total flange thickness $t_{\mathrm{T}}$ was taken to be the manufacture's specified thickness. For the sensitivity analysis, $c$ was varied between values based on those measured on the real specimens. The wrinkle amplitude $A_{\mathrm{w}}$ was varied from $0 \mathrm{~mm}$ to the maximum practical amplitude of $6.5 \mathrm{~mm}$, where the top fibre mat dips down and touches the bottom fibre mat. Note that this amplitude is over three times greater than the maximum measured amplitude of $2.0 \mathrm{~mm}$ shown in Figure 16(c).

Both the elastic properties of the top flange and the failure load were determined using the approach outlined in Bogetti et al. [23], which is based on dividing the model into discrete segments $\mathrm{d} x$ and systematically applying 2D classical laminate theory to each segment (Figure 19(b)). The elastic and strength properties of each lamina were taken from those reported in a previous study [28] for a similar pultruded GFRP material, and are given in Table 2. The average axial and bending stiffnesses were obtained by integrating the laminate stiffness matrices of each differential segment over the total length $L$. A quadratic failure criterion was used to determine intralaminar failure for each $\mathrm{d} x$ segment within the laminate. Additionally, interlaminar failure between the wrinkled mat-to-roving interface was considered by applying a weakening factor of 0.3 to the normal and shear strengths of the mat layer. This 
weakening factor was derived in [28] for a FE model of a similar pultruded GFRP material using a sensitivity analysis. A uniaxial tensile load was considered in this analysis as this partially reflects the stress state at the near surface wrinkles present in the pultruded deck under tyre loading. Under a tensile load the wrinkled mat layer will develop local out-of-plane normal $\sigma_{3}$ and interlaminar shear $\tau_{13}$ stresses due to the misalignment angle $\theta$. These stresses are calculated by tensorial transformation of the $x$ direction stress $\sigma_{x}$.

\subsection{Results and discussion}

Figure 20(a) and (b) show the reduction in axial and bending stiffnesses as a function of wrinkle amplitude $A_{\mathrm{w}}$, respectively. The stiffnesses are normalised relative to the values for $A_{\mathrm{w}}=0$. In each figure, two curves are shown for different wrinkle widths of $c=2.0 \mathrm{~mm}$ and $c=5.0 \mathrm{~mm}$, and the area less than $A_{\mathrm{w}}=2.0$ is shaded in grey to reflect the maximum range of measured wrinkle amplitude in the bridge deck specimens. From these figures the following conclusions can be drawn:

- The maximum practical wrinkle amplitude of $6.3 \mathrm{~mm}$ results in a reduction in axial and bending stiffnesses of $13 \%$ and $20 \%$, respectively. As expected, the influence on bending stiffness is greater than that on axial stiffness due to the reduction in second moment of area as a consequence of the wrinkled fibre mat being closer to the flange mid-surface.

- At the maximum measured amplitude of $2.0 \mathrm{~mm}$, the axial and bending stiffnesses reduced by $4.8 \%$ and $7.5 \%$ respectively. However, at this amplitude, a smaller $c$ value of $2.0 \mathrm{~mm}$ produced a greater reduction in stiffness. The cross-over point of the two curves for $c=2.0 \mathrm{~mm}$ and $c=5.0$ $\mathrm{mm}$ occurs because a narrower wrinkle produces a greater misalignment angle but over a smaller length of flange compared to a wide wrinkle.

In Figure 21, the normalised knockdown tensile load - defined as the tensile failure load divided by the failure load at $A_{\mathrm{w}}=0$ - is plotted as a function of wrinkle amplitude for two different wrinkle widths of $c=2.0 \mathrm{~mm}$ and $c=5.0 \mathrm{~mm}$, for an applied uniaxial tensile load. For $A_{\mathrm{w}}=0$, failure manifests as tensile failure in the mat layers. However, as the wrinkle amplitude is increased, the interlaminar normal and shear stress demand increases, resulting in interlaminar failure at the mat-roving interface. This transition point occurs at $A_{\mathrm{w}}=0.3 \mathrm{~mm}$ and $A_{\mathrm{w}}=0.8 \mathrm{~mm}$ for values of $c=2.0 \mathrm{~mm}$ and $c=5.0 \mathrm{~mm}$, respectively. 

previously in Figure 16, which had $A_{\mathrm{w}}=2.0 \mathrm{~mm}, c=4.7 \mathrm{~mm}$ for specimen DT and $A_{\mathrm{w}}=1.5 \mathrm{~mm}, c=2.8$ $\mathrm{mm}$ for specimen $\mathrm{BB}$, are $53 \%$ and $57 \%$, respectively. The greater load capacity reduction in BB is due to the fact that the wrinkle has a higher maximum tangent angle and thus a higher interlaminar stress demand, despite the wrinkle having a smaller amplitude.

An important feature of Figure 21 is the sensitivity of the failure load to small changes in wrinkle amplitude once interlaminar failure becomes the limiting criterion. For example, for a wrinkle width of $c=2.0 \mathrm{~mm}$, starting at $A_{\mathrm{w}}=0.3 \mathrm{~mm}$ a $100 \%$ increase in $A_{\mathrm{w}}$ leads to a $40 \%$ drop in failure load. This sensitivity suggests that the manufacturing tolerance for wrinkle amplitude should be well below that which leads to interlaminar failure, which for small wrinkle widths is approximately $A_{\mathrm{w}}=0.3 \mathrm{~mm}$.

\section{Conclusions and future work}

The following conclusions can be drawn from this study:

- The fibre mats observed in a specific pultruded, cellular GFRP bridge deck exhibited different classes of misalignment in the transverse-to-pultrusion direction, each of which had a distinct morphology. Six different classes of mat misalignment were distinguished: double-backs, flips, near-surface wrinkles, corner wrinkles, waviness and drops.

- Double-backs, flips and corner wrinkles arose due to errors during the wrapping of the mats around the internal mandrel, near-surface wrinkles were caused by resin injection, waviness from shifting of the longitudinal rovings adjacent to the mats, and drops from the gaps between the internal mats.

- The mat misalignments were inconsistent between nominally identical WFJs taken from the different specimens of the same decking system. Out of the ten WFJs analysed, double backs occurred only in one, flips in three and wrinkles in four of the WFJs. The length, height and steepest tangent angle of the wavy mat layer passing through each junction were highly variable, as was the distance from the centre of the junctions at which the different mat layers were terminated. 
- Extreme forms of mat misalignment, for example double backs and flips, can result in displacement of the mandrels during pultrusion due to unbalanced compression forces from the fibres. Mandrel displacement resulted in variable flange thicknesses either side of the WFJs.

- Mat misalignments are not solely a feature of the WFJs. Near-surface wrinkles near the local flange midspan were more severe than those in the WFJs. Predictions based on classical laminate theory showed that the near surface wrinkles observed in the flanges led to a laminate tensile load capacity reduction of up to $57 \%$ due to the increased interlaminar stress demand and the point of steepest tangent angle at the fibre mat-to-roving interface.

The taxonomy of fibre mat misalignments provided in this paper can be readily adopted by pultruders as a framework for improving the nature and consistency of mat misalignments within cellular profiles. In particular, the different misalignment categories outlined in this paper have been intentionally linked to a specific aspect of the pultrusion process so that remediation measures can be targeted appropriately. For example, the near-surface wrinkles observed at the junctions and local flange midspans may be minimised or relocated through better control of the resin injection pressure within the die.

Currently, this defect occurs in areas of maximum local bending and shear when the deck is subjected to the local contact pressure distribution of heavy lorry tyres, and hence may be compromising the deck's long-term performance. Furthermore, the optical technique for measuring and characterising the severity of mat misalignment can, with further research into the underlying mechanics, be implemented into existing quality standards for pultruded profiles.

To further substantiate and extend the practical implications of the findings presented in this paper, future work should use experimental observations of fracture patterns developed during both quasistatic and fatigue loading of the deck to establish which categories of misalignment are most significant to structural performance. This can be supplemented with data from FE simulations that independently vary the severity of each type of mat misalignment identified in this paper. Furthermore, the variability of mat misalignments should be assessed in the direction of pultrusion by making multiple section cuts along a representative length of deck, although such longitudinal misalignments are expected to be much less owing to the longitudinal tension exerted on the associated fibres during the pultrusion manufacturing process. 


\section{References}

[1] Hollaway LC. A review of the present and future utilisation of FRP composites in the civil infrastructure with reference to their important in-service properties. Constr Build Mater $2010 ; 24: 2419-45$.

[2] Sebastian WM. Fibre waviness in pultruded bridge deck profiles: Geometric characterisation and consequences on ultimate behaviour. Compos Part B Eng 2018;146:270-80.

[3] Sebastian WM, Ralph M, Poulton M, Goacher J. Lab and field studies into effectiveness of flat steel plate - rubber pad systems as tyre substitutes for local loading of cellular GFRP bridge decking. Compos Part B Eng 2017;125:100-22.

[4] ASTM International. D4385-19 Standard Practice for Classifying Visual Defects in Thermosetting Reinforced Plastic Pultruded Products. West Conshohocken (PA): 2019.

[5] British Standards Institute. BS EN 13706-2:2002, Reinforced plastics composites. Specifications for pultruded profiles. Method of test and general requirements. 2002.

[6] Coogler K, Rizos DC, Wan B, Harries KA, Petrou MF. Critical Evaluation of Strain Measurements in Glass Fiber-Reinforced Polymer Bridge Decks. J Bridg Eng 2005;10:704-12.

[7] Yanes-Armas S, de Castro J, Keller T. Energy dissipation and recovery in web-flange junctions of pultruded GFRP decks. Compos Struct 2016;148:168-80.

[8] Xin H, Mosallam AS, Liu Y, Wang C, He J. Experimental and numerical investigation on assessing local bearing behavior of a pultruded GFRP bridge deck. Compos Struct 2018;204:71230.

[9] Sá MF, Correia JR, Silvestre N, Gomes AM. Transverse bending and in-plane shear behaviours of multicellular pultruded GFRP deck panels with snap-fit connections. Thin-Walled Struct $2020 ; 154$.

[10] Wang Y, Zureick AH. Characterization of the longitudinal tensile behavior of pultruded I-shape structural members using coupon specimens. Compos Struct 1994;29:463-72.

[11] Bank LC, Yin J, Nadipelli M. Local buckling of pultruded beams - nonlinearity, anisotropy and inhomogeneity. Constr Build Mater 1995;9:325-31.

[12] Turvey GJ, Zhang Y. Characterisation of the rotational stiffness and strength of web-flange junctions of pultruded GRP WF-sections via web bending tests. Compos Part A Appl Sci Manuf 
2006;37:152-64.

[13] Liu TQ, Yang JQ, Feng P, Harries KA. Determining rotational stiffness of flange-web junction of pultruded GFRP I-sections. Compos Struct 2020;236:111843.

[14] Liu T, Harries KA. Flange local buckling of pultruded GFRP box beams. Compos Struct 2018;189:463-72.

[15] Mottram JT. Reference and Bibliography Database on Research and Development with Pultruded Fibre Reinforced Polymer Shapes and Systems 2020 https://warwick.ac.uk/fac/sci/eng/staff/jtm/pfrp_latest.pdf (accessed November 20, 2020).

[16] Shaw-Stewart D, Sumerak J. The Pultrusion Process. In: Starr T, editor. Pultrusion Eng., Cambridge: Woodhead Publishing Limited; 2000, p. 19-65.

[17] Meyer R. Handbook of Pultrusion Technology. New York: Chapman and Hall; 1985.

[18] Kulkarni P, Mali KD, Singh S. An overview of the formation of fibre waviness and its effect on the mechanical performance of fibre reinforced polymer composites. Compos Part A Appl Sci Manuf 2020;137:106013.

[19] El-Hajjar RF, Petersen DR. Gaussian function characterization of unnotched tension behavior in a carbon/epoxy composite containing localized fiber waviness. Compos Struct 2011;93:2400-8.

[20] Adams DOH, Hyer MW. Effect of Layer Waviness on the Compression Strength of Thermoplastic Composte Laminates. J Reinf Plast Compos 1993;12:414-29.

[21] Wang J, Potter KD, Hazra K, Wisnom MR. Experimental fabrication and characterization of outof-plane fiber waviness in continuous fiber-reinforced composites. J Compos Mater 2012;46:2041-53.

[22] Potter K, Khan B, Wisnom M, Bell T, Stevens J. Variability, fibre waviness and misalignment in the determination of the properties of composite materials and structures. Compos Part A Appl Sci Manuf 2008;39:1343-54.

[23] Bogetti TA, Gillespie JW, Lamontia MA. The Influence of Ply Waviness with Nonlinear Shear on the Stiffness and Strength Reduction of Composite Laminates. J Thermoplast Compos Mater 1994;7:76-90.

[24] Hsiao HM, Daniel IM. Elastic properties of composites with fiber waviness. Compos Part A Appl Sci Manuf 1996;27(10):931-41. 
[25] Hsiao HM, Daniel IM. Effect of Fibre Waviness on Stiffness and Strength Reduction of Unidirectional Composites under Compressive Loading. Compos Sci Technol 1996;56(5):58193.

[26] Sobek W, Trumpf H. The new lightweight bridge deck FBD300 made from pultruded fiberreinforced composites for traffic bridges. Proc 4th Int Conf FRP Compos Civ Eng CICE 2008 2008:22-4.

[27] Baran I, Straumit I, Shishkina O, Lomov S V. X-ray computed tomography characterization of manufacturing induced defects in a glass/polyester pultruded profile. Compos Struct 2018;195:74-82.

[28] Girão Coelho AM, Mottram JT, Matharu N. Virtual characterization of delamination failures in pultruded GFRP angles. Compos Part B Eng 2016;90:212-22. 